\title{
Mitotic/Spindle Checkpoint
}

National Cancer Institute

\section{Source}

National Cancer Institute. Mitotic/Spindle Checkpoint. NCI Thesaurus. Code C21492.

The mitotic/spindle checkpoint ensures that all replicated chromatids are properly connected to opposing spindle poles before separation (anaphase) can begin. The proteins responsible for this form a complex on the kinetochores. 